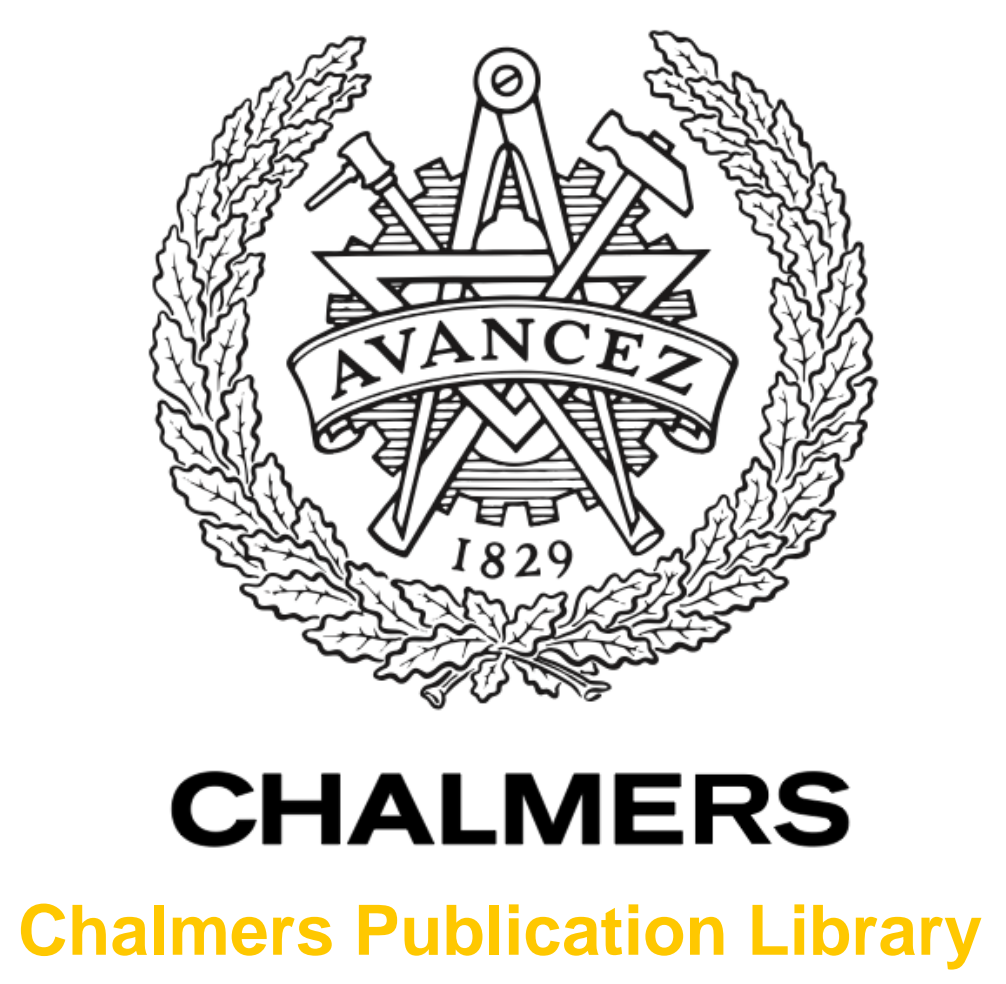

Minimum Common Outage Probability for the Broadcast Relay Channel

This document has been downloaded from Chalmers Publication Library (CPL). It is the author's version of a work that was accepted for publication in:

\author{
IWCMC 2011 - 7th International Wireless Communications and Mobile Computing \\ Conference. Istanbul, 4-8 July 2011 \\ Citation for the published paper: \\ Isikman, A. ; Yuksel, M. (2011) "Minimum Common Outage Probability for the Broadcast \\ Relay Channel". IWCMC 2011 - 7th International Wireless Communications and Mobile \\ Computing Conference. Istanbul, 4-8 July 2011 pp. 894-899.
}

Downloaded from: http://publications.lib.chalmers.se/publication/157564

Notice: Changes introduced as a result of publishing processes such as copy-editing and formatting may not be reflected in this document. For a definitive version of this work, please refer to the published source. Please note that access to the published version might require a subscription. 


\section{Minimum Common Outage Probability for the Broadcast Relay Channel}

\author{
Arif Önder Isıkman \\ Department of Signals and Systems \\ Chalmers University of Technology \\ Gothenburg, Sweden \\ isikman@student. chalmers.se
}

\author{
Melda Yuksel \\ EEE Department \\ TOBB University of Economics and Technology \\ Ankara, Turkey \\ yukseldetu.edu.tr
}

\begin{abstract}
In this paper the broadcast relay channel (BRC) is studied. In the BRC model, the source communicates with two destinations with the help of a single relay. The minimum common outage probability for four different transmission protocols, direct transmission (DT), multihop (MH), path selection (PS) and link combination with path selection (LCPS) is investigated under long-term power constraint for constant rate transmission. In addition, the $\epsilon$-outage rate region for a fixed common outage probability is computed. Based on the cut-set bound (CSB), a lower bound on the minimum common outage probability and an upper bound on the $\epsilon$-outage rate region are also found. Numerical results suggest that enforcing the relay to help both destinations simultaneously is limiting. The dominant factor in the gains obtained with respect to DT is due to path selection and link combination is not necessary when the relay is close to the source.
\end{abstract}

Index Terms - broadcast relay channel, common outage probability, cooperation, power allocation, long-term power constraint, wireless channels

\section{INTRODUCTION}

The relay channel was introduced more than thirty years ago [1]; however, it was not until [2] and [3] that research on the relay channel picked up that proved that relaying can enhance reliability in wireless channels. Similar to multiple antenna techniques, relaying schemes are viable methods that mitigate the adverse effects of fading [2], [3]. The gains cooperation/relaying promises in wireless channels have resulted in a significant expansion in the literature over the last decade (see [4], [5], [6] and references therein.)

The four-terminal broadcast relay channel (BRC) is first studied in [7]. In the BRC model, there are two destinations, which communicate with the source with the help of a single dedicated relay. The capacity region of the BRC, when both receivers are degraded with respect to the relay is found in [8]. This result is extended to Gaussian channels in [8], [9]. An achievable rate region for the K-receiver broadcast channel with a single relay is investigated in [10].

When channel state information is available at the transmitter, substantial gains can be achieved if transmission power is adapted according to the channel conditions. In [11], an optimal constant-rate transmission scheme for the block fading

${ }^{1}$ This material is based upon work supported by the Scientific and Technological Research Council of Turkey, TUBITAK, under Grant No. 108E208. point-to-point channel is found. Under a long-term power constraint, it is proved that the optimal power allocation scheme that minimizes outage probability is of thresholdtype [11]. Generalizing the single-user results, an optimal power allocation protocol and the resulting capacity region are established in [12] for parallel Gaussian broadcast channels. Optimal power allocation protocols for minimum outage probability for fading broadcast channels and multi-hop relay channels are studied in [13] and [14]. Under long-term power constraint, upper and lower bounds on the outage capacity of the fading relay channel are found in [15]. Outage probability for the relay channel under long-term power constraint is also studied in [16]. In [16], opportunistic protocols, in which the relay is not utilized if cooperation consumes more power with respect to direct transmission are proposed and proved to perform close to the cut-set bound. For fading relay channels, power allocation methods that maximize achievable rates are obtained in [17].

In this paper we investigate relaying strategies and related power allocation methods that minimize outage probability for the BRC. Assuming long-term power constraint and constantrate transmission, we study the common outage probability for both of the destinations. The common outage probability is introduced in [13] as a relevant performance measure for broadcast channels. In a broadcast channel, depending on the channel state, the broadcast channel can either not be used at all, or transmission to both of the receivers take place at the same time. Such an operation mode, which is different from declaring outage for each of the receivers individually, is necessary if coordination among receivers is to be established.

In the BRC setting, we study the minimum common outage probability for a fixed rate pair and the $\epsilon$-outage rate region for a fixed common outage probability for four different protocols: direct transmission (DT), multihop (MH), path selection (PS) and link combination with path selection (LCPS). In all of these protocols, the source uses superposition coding for the two independent messages it has for each of the destinations. The DT protocol serves as a benchmark to show the benefits of relaying. In $\mathrm{MH}$, the relay has to decode both messages to help both destinations and the destinations only listen to the relay. However, in PS, we exploit the superposition of messages, allow for the relay to help only one of the destinations and let 


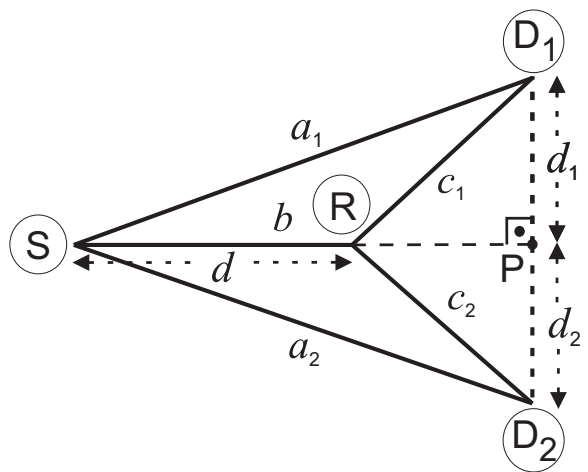

Fig. 1. The broadcast relay channel, (BRC) with one source (S), one relay (R), and two destinations $\left(\mathrm{D}_{1}, \mathrm{D}_{2}\right)$. For the numerical simulations in Section $\mathrm{IV}$, all four nodes are located on a plane with $\mathrm{S}-\mathrm{R}, \mathrm{D}_{1}$-point $\mathrm{P}$ and $\mathrm{D}_{2}$-point $\mathrm{P}$ distances are respectively equal to $d, d_{1}$ and $d_{2}$.

the other destination directly listen to the source. In this case, the destination that listens to the relay does not listen to the source. In LCPS, we explore the gains introduced when the destinations can combine the signals both from the source and the relay. Finally, we compare all these four protocols with the cut-set bound (CSB). Our results indicate that path selection is the dominant factor in the gains obtained and the gains due to link combination are insignificant, when the relay is close to the source. However, the gains due to link combination are emphasized when the relay is far from the source.

The organization of the rest of the paper is as follows. In Section II, the system model is introduced. In Section III, the transmission protocols are described in detail. In Section IV, the common outage probability results are presented. Finally in Section V, the paper is concluded.

\section{System ModeL}

The BRC consists of one source (S), one relay (R), and two destinations $\left(D_{1}, D_{2}\right)$ as shown in Fig. 1. The instantaneous amplitude squares of complex channel gains among $S-D_{1}, S-$ $\mathrm{D}_{2}, \mathrm{~S}-\mathrm{R}, \mathrm{R}-\mathrm{D}_{1}$ and $\mathrm{R}-\mathrm{D}_{2}$ are respectively denoted by $a_{1}, a_{2}$, $b, c_{1}$ and $c_{2}$. It is assumed that the channel gain amplitudes are known globally at all nodes, whereas channel gain phases are known only at corresponding receivers. The channel coefficients have quasi-static fading [11] and are independent from one block to the other. Complex Gaussian noise at the receivers are independent, and have zero mean and unit variance.

For the numerical results we present in Section IV, we assume $a_{1}, a_{2}, b, c_{1}$, and $c_{2}$ are independent exponential random variables and we assume all terminals are located on a plane. The relay is located on the line joining the source and point $\mathrm{P}$, where $\mathrm{S}-\mathrm{R}$ distance is $d$ and the distance between the source and point $\mathrm{P}$ is normalized to $1 . \mathrm{D}_{1}$ $\mathrm{P}$ and $\mathrm{D}_{2}-\mathrm{P}$ distances are respectively denoted as $d_{1}$ and $d_{2}$. As a result, the random variables $a_{1}, a_{2}, b, c_{1}$, and $c_{2}$ respectively have the mean values $\left(1+d_{1}^{2}\right)^{-\frac{\alpha}{2}},\left(1+d_{2}^{2}\right)^{-\frac{\alpha}{2}}$, $d^{-\alpha},\left[(1-d)^{2}+d_{1}^{2}\right]^{-\frac{\alpha}{2}},\left[(1-d)^{2}+d_{2}^{2}\right]^{-\frac{\alpha}{2}}$, where $\alpha$ is the path loss exponent.

We assume the relay is half-duplex and there is time division among the source and the relay. The source transmits for $t$ fraction of the block, $0<t \leq 1$, and the relay transmits in the rest $1-t$.

The source node has two independent messages $W_{1}$ and $W_{2}$ at target rates $R_{1}$ and $R_{2}$ for each of the destinations. The source node encodes $W_{1}$ and $W_{2}$ into $X_{1}$ and $X_{2}$ using superposition coding [18]. It allocates power $P_{S 1}^{(i)}(s, t)$ to send $X_{1}, P_{S 2}^{(i)}(s, t)$ to send $X_{2}$, and transmits $X=X_{1}+X_{2}$ to reach both destinations simultaneously at channel state $s=$ $\left(a_{1}, a_{2}, b, c_{1}, c_{2}\right)$, for a fixed $t$, for protocol $i, i=\mathrm{DT}, \mathrm{MH}, \mathrm{PS}$, LCPS. Upon receiving the source signal $X$, the relay decodes $X_{1}, X_{2}$ or both depending on the transmission protocol. The relay allocates power $P_{R 1}^{(i)}(s, t)$ to forward $W_{1}$ to $\mathrm{D}_{1}$, and $P_{R 2}^{(i)}(s, t)$ to forward $W_{2}$ to $\mathrm{D}_{2}$. We assume the source and the relay have a long-term average total power constraint $P_{\text {avg }}$ :

$$
\begin{aligned}
\int_{s}[t & \left(P_{S 1}^{(i)}(s, t)+P_{S 2}^{(i)}(s, t)\right)+(1-t) \\
& \left.\times\left(P_{R 1}(s, t)+P_{R 2}(s, t)\right)\right] f(s) \mathrm{d} s \leq P_{a v g}
\end{aligned}
$$

where $f(s)$ denotes the probability density function of the channel state $s$.

Let $\left(\hat{P}_{S 1}^{(i)}(s, t), \hat{P}_{S 2}^{(i)}(s, t), \hat{P}_{R 1}^{(i)}(s, t), \hat{P}_{R 2}^{(i)}(s, t)\right)$ denote the minimum amount of power levels that satisfy the condition $C_{j}^{(i)} \geq R_{j}, i=\mathrm{DT}, \mathrm{MH}, \mathrm{PS}, \mathrm{LCPS}, j=1,2$, for a fixed $t$, where $C_{j}^{(i)}$ denotes the achievable rate at $j$ th destination with protocol $i$. Then the minimum total amount of power required for reliable communication for protocol $i$, at state $s$ is given as 1

$$
\begin{aligned}
P_{r e q}^{(i)}(s)= & \min _{t} t\left(\hat{P}_{S 1}^{(i)}(s, t)+\hat{P}_{S 2}^{(i)}(s, t)\right) \\
& +(1-t)\left(\hat{P}_{R 1}^{(i)}(s, t)+\hat{P}_{R 2}^{(i)}(s, t)\right), \\
= & t^{*}\left(\hat{P}_{S 1}^{(i)}\left(s, t^{*}\right)+\hat{P}_{S 2}^{(i)}\left(s, t^{*}\right)\right) \\
& +\left(1-t^{*}\right)\left(\hat{P}_{R 1}^{(i)}\left(s, t^{*}\right)+\hat{P}_{R 2}^{(i)}\left(s, t^{*}\right)\right),
\end{aligned}
$$

where $t^{*}$ is the best $t, 0<t \leq 1$ that minimizes (2). Note that the fraction of the time the relay listens, $t$, is a function of the channel state vector $s$.

Given $P_{r e q}^{(i)}(s)$ for the $i$ th protocol at state $s$, the optimal resource allocation function that attains the minimum common outage probability is of the threshold type [11], [13], [16]. As a result $P_{S 1}^{(i)}\left(s, t^{*}\right), P_{S 2}^{(i)}\left(s, t^{*}\right), P_{R 1}^{(i)}\left(s, t^{*}\right)$ and $P_{R 2}^{(i)}\left(s, t^{*}\right)$ are determined according to

$$
P_{k j}^{(i)}\left(s, t^{*}\right)=\left\{\begin{array}{lll}
0 & \text { if } & P_{r e q}^{(i)}(s) \geq P_{t h} \\
\hat{P}_{k j}^{(i)}\left(s, t^{*}\right) & \text { if } & P_{r e q}^{(i)}(s)<P_{t h}
\end{array}\right.
$$

for $k=\mathrm{S}, \mathrm{R}, j=1,2$, where $P_{t h}$ is determined such that (1) is satisfied. ${ }^{2}$

This threshold type behavior can be explained as follows: For very poor channel conditions, the total power level required to send the target rates $R_{1}$ and $R_{2}$ respectively to $\mathrm{D}_{1}$

${ }^{1}$ For MH, PS and LCPS protocols, different operation modes, DF, RH1 and $\mathrm{RH} 2$, will be defined in the next section. This definition also applies to $i=$ DF, RH1, RH2.

${ }^{2}$ From this point on, we will denote $\hat{P}_{k j}^{(i)}(s, t)$ and $P_{k j}^{(i)}(s, t)$ respectively with $\hat{P}_{k j}^{(i)}$ and $P_{k j}^{(i)}$ for a simpler notation. 
and $\mathrm{D}_{2}$ is very high. If transmission is sustained during such poor channel conditions, power is wasted to invert the channel and the total average power constraint in (1) is violated. However, if transmission is discontinued until the channel conditions become favorable, then power can be saved and (1) is satisfied. For those channel states during which transmission is off, or when $P_{r e q}^{(i)}(s) \geq P_{t h}$, the system is in outage. Subject to the total average power constraint $P_{a v g}$, one can determine $P_{t h}$ such that the common outage probability for protocol $i$

$$
P_{\text {out }}^{(i)}=\mathbb{P}\left(P_{\text {req }}^{(i)}(s) \geq P_{\text {th }}\right)
$$

is minimized. Then for a given target rate pair $\left(R_{1}, R_{2}\right)$, the minimum common outage probability is given as

$$
P_{\text {out-min }}^{(i)}=\min P_{\text {out }}^{(i)} \quad \text { subject to (1). }
$$

In this paper our objective is to find the minimum common outage probability defined in (6). We investigate four protocols DT, MH, PS, and LCPS in comparison to the cut-set bound (CSB). In each of these cases, $\mathrm{D}_{1}$ and $\mathrm{D}_{2}$ are turned on or off simultaneously. In addition to minimum common outage probability for a given rate pair $\left(R_{1}, R_{2}\right)$, we also analyze the $\epsilon$-outage rate region. The $\epsilon$-outage rate region is the collection of all achievable rate pairs, for which the common outage probability (5) is at most $\epsilon$ and the total average power constraint (1) is satisfied. Note that the minimum common outage probability problem of (6) and the $\epsilon$-outage rate region problem are inherently the same. We refer the reader to [13] for the proof and omit the details here.

\section{TRANSMISSION PROTOCOLS}

In this section we describe the protocols DT, MH, PS and LCPS and CSB, in detail and find the amount of power needed $\left(\hat{P}_{S 1}^{(i)}, \hat{P}_{S 2}^{(i)}, \hat{P}_{R 1}^{(i)}, \hat{P}_{R 2}^{(i)}\right), i=\mathrm{DT}, \mathrm{MH}, \mathrm{PS}, \mathrm{LCPS}, \mathrm{CSB}$ for reliable communication for each channel state $s$ and for a fixed $t$.

\section{A. Direct Transmission}

In DT, the relay is not utilized and the system is equivalent to a broadcast channel. The source node transmits all the time, $t=1$. The minimum common outage probability for the broadcast channel is solved in [13]. Here, we restate $\hat{P}_{S 1}^{(D T)}$ and $\hat{P}_{S 2}^{(D T)}$ as DT is a part of all other protocols under study.

When $a_{1} \geq a_{2}$ and $P_{S 1}$ and $P_{S 2}$ are the power levels allocated to $X_{1}$ and $X_{2}$ at the source, the best rate pair (the rate pair on the capacity region of the broadcast channel) is

$$
\begin{aligned}
& C_{S 1}^{(D T)}=\log \left(1+a_{1} P_{S 1}\right) \\
& C_{S 2}^{(D T)}=\log \left(1+\frac{a_{2} P_{S 2}}{a_{2} P_{S 1}+1}\right) .
\end{aligned}
$$

When $a_{1}<a_{2}$, the subscripts " 1 " and " 2 " need to be interchanged in (7) and (8). Solving $C_{S 1}^{(D T)}=R_{1}$ and $C_{S 2}^{(D T)}=R_{2}$ for $P_{S 1}$ and $P_{S 2}$, one can calculate the minimum amount of power required $\left(\hat{P}_{S 1}^{(D T)}\right.$ and $\left.\hat{P}_{S 2}^{(D T)}\right)$ to attain a given rate $R_{1}$ at

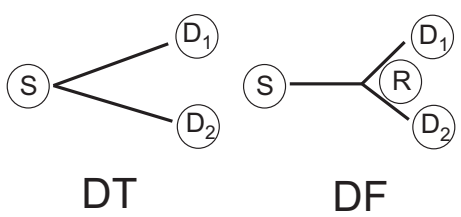

Fig. 2. Operation modes for MH: direct transmission (DT) and decode-andforward (DF). The destinations do not listen to the source in DF mode.

the first destination, and $R_{2}$ at the second destination. Defining $g$ as a function with three inputs and two outputs as

$$
\begin{aligned}
\left(y_{1}, y_{2}\right) & \triangleq g\left(x_{1}, x_{2}, x_{3}\right) \\
y_{1} & =\left\{\begin{array}{lll}
\left(2^{\frac{R_{1}}{x_{3}}}-1\right) \frac{1}{x_{1}} & \text { if } & x_{1} \geq x_{2} \\
\left(2^{\frac{R_{1}}{x_{3}}}-1\right)\left(\frac{1}{x_{1}}+y_{2}\right) & \text { if } & x_{1}<x_{2}
\end{array}\right. \\
y_{2} & =\left\{\begin{array}{lll}
\left(2^{\frac{R_{2}}{x_{3}}}-1\right)\left(\frac{1}{x_{2}}+y_{1}\right) & \text { if } & x_{1} \geq x_{2} \\
\left(2^{\frac{R_{2}}{x_{3}}}-1\right) \frac{1}{x_{2}} & \text { if } & x_{1}<x_{2}
\end{array}\right.
\end{aligned}
$$

$\hat{P}_{S 1}^{(D T)}$ and $\hat{P}_{S 2}^{(D T)}$ become $\left(\hat{P}_{S 1}^{(D T)}, \hat{P}_{S 2}^{(D T)}\right)=g\left(a_{1}, a_{2}, 1\right)$. Then, $P_{r e q}^{(D T)}(s)$ can be calculated from (3), leading to the minimum common outage probability calculation in (6).

\section{B. Multihop}

In $\mathrm{MH}$, there are two modes of operation: direct transmission (DT) and decode-and-forward (DF). The two modes of operation for $\mathrm{MH}$ are shown in Fig. 2.

Consider the three terminal relay channel with direct link gain, $a$, source-relay link gain $b$ and relay-destination gain $c$. If $a<c$ and $a<b$, it is shown in [16] that transmission over the multihop route via the relay consumes less power than sending the message directly to the destination. Motivated by this result, we assume the source aims to reach both $D_{1}$ and $\mathrm{D}_{2}$ via the relay if $s \in \boldsymbol{A}_{M H}^{D F}$ where

$$
\boldsymbol{A}_{M H}^{D F}=\left\{s:\left(a_{1}<c_{1} \cap a_{1}<b\right) \cap\left(a_{2}<c_{2} \cap a_{2}<b\right)\right\} .
$$

In this case, we say the system is in DF mode. In DF mode, in order for the relay to decode both messages reliably, the required power levels at the source are

$$
\left(\hat{P}_{S 1}^{(D F)}, \hat{P}_{S 2}^{(D F)}\right)=g(b, b, t) .
$$

Using independent codebooks, the relay then reencodes $W_{1}$ and $W_{2}$, and forwards them to the destinations using superposition coding. As the destinations only listen to relay, the relay power needed for reliable reception at the destinations is

$$
\left(\hat{P}_{R 1}^{(D F)}, \hat{P}_{R 2}^{(D F)}\right)=g\left(c_{1}, c_{2}, 1-t\right) .
$$

If $s \in \boldsymbol{A}_{M H}^{D T}$, where $\boldsymbol{A}_{M H}^{D T}=\left(\boldsymbol{A}_{M H}^{D F}\right)^{c}$ and $c$ denotes the complement, we assume the relay is not utilized and the system operates in DT mode. Then

$$
P_{r e q}^{(M H)}(s)=\left\{\begin{array}{ccc}
P_{r e q}^{(D F)}(s) & \text { if } & s \in \boldsymbol{A}_{M H}^{D F} \\
P_{r e q}^{(D T)}(s) & \text { if } & s \in \boldsymbol{A}_{M H}^{D T}
\end{array}\right.
$$


Using (13), the minimum common outage probability of (6)is then calculated.

\section{Path Selection}

In the PS protocol, there are four modes of operation, DT, relay helps user 1 (RH1), relay helps user 2 (RH2) and DF, which are shown in Fig. 3. Unlike MH, in PS the relay is not required to decode both messages $W_{1}$ and $W_{2}$, but can decode only one of them depending on the channel state $s$. This decreases the decoding constraint at the relay, and increases its chance to be more useful for one of the destinations.

The system is in RH1 mode if $s \in \boldsymbol{A}_{P S}^{R H 1}$ where

$$
\boldsymbol{A}_{P S}^{R H 1}=\left\{s:\left(a_{1}<c_{1} \cap a_{1}<b\right) \cap\left(a_{2}>c_{2} \cup a_{2}>b\right)\right\} .
$$

In $\mathrm{RH} 1$, the source transmits to the relay and to $\mathrm{D}_{2}$ using superposition coding. $\mathrm{D}_{1}$ does not listen to the source, but only to the relay. This is a practical assumption that enables simple receivers. In RH1, the relay is only required to decode $W_{1}$. The relay and $\mathrm{D}_{2}$ can respectively decode $W_{1}$ and $W_{2}$ reliably if $\left(\hat{P}_{S 1}^{(R H 1)}, \hat{P}_{S 2}^{(R H 1)}\right)=g\left(b, a_{2}, t\right)$. Upon decoding $W_{1}$, the relay independently reencodes and forwards the message to $\mathrm{D}_{1}$ with

$$
\left(\hat{P}_{R 1}^{(R H 1)}, \hat{P}_{R 2}^{(R H 1)}\right)=\left[\left(2^{\frac{R_{1}}{1-t}}-1\right) \frac{1}{c_{1}}, 0\right] .
$$

Note that $\mathrm{D}_{2}$ does not need to listen to the relay, as the relay's transmission carries information only about $W_{1}$ and is of no use at $\mathrm{D}_{2}$.

The RH2 mode is similar to RH1 and the relay only assists $\mathrm{D}_{2}$. The system is in RH2 mode if $s \in \boldsymbol{A}_{P S}^{R H^{2}}$, where $\boldsymbol{A}_{P S}^{R H^{2}}=$ $\left\{s:\left(a_{1}>c_{1} \cup a_{1}>b\right) \cap\left(a_{2}<c_{2} \cap a_{2}<b\right)\right\}$. In RH2, the required source and relay power levels are given as

$$
\begin{aligned}
& \left(\hat{P}_{S 1}^{(R H 2)}, \hat{P}_{S 2}^{(R H 2)}\right)=g\left(a_{1}, b, t\right) \\
& \left(\hat{P}_{R 1}^{(R H 2)}, \hat{P}_{R 2}^{(R H 2)}\right)=\left[0,\left(2^{\frac{R_{2}}{1-t}}-1\right) \frac{1}{c_{2}}\right] .
\end{aligned}
$$

In PS, if $s \in \boldsymbol{A}_{P S}^{D F}$, where $\boldsymbol{A}_{P S}^{D F}=\boldsymbol{A}_{M H}^{D F}$ defined in (10), then the system is in DF mode. The relay decodes both $W_{1}$ and $W_{2}$ and forwards these messages to $\mathrm{D}_{1}$ and $\mathrm{D}_{2}$. The required source and relay power levels are given in (11) and (12).

Finally, we assume the system is in DT mode if it is not in anyone of the above defined operation modes. Then

$$
P_{r e q}^{(P S)}(s)=\left\{\begin{array}{lll}
P_{r e q}^{(R H 1)}(s) & \text { if } & s \in \boldsymbol{A}_{P S}^{R H 1} \\
P_{r e q}^{(R H 2)}(s) & \text { if } & s \in \boldsymbol{A}_{P S}^{R H 2} \\
P_{r e q}^{(D F)}(s) & \text { if } & s \in \boldsymbol{A}_{P S}^{D F} \\
P_{r e q}^{(D T)}(s) & \text { if } & s \in \boldsymbol{A}_{P S}^{D T}
\end{array}\right.
$$

Finally, (18) is used to calculate the minimum common outage probability of (6).

\section{Link Combination with Path Selection}

In LCPS, the operation modes are the same as PS. However, in LCPS the destinations listen to the signals from both the source and the relay. Although, LCPS is sure to perform better than PS, it requires complex receivers and its use is limited. An illustration of LCPS is shown in Fig. 3.

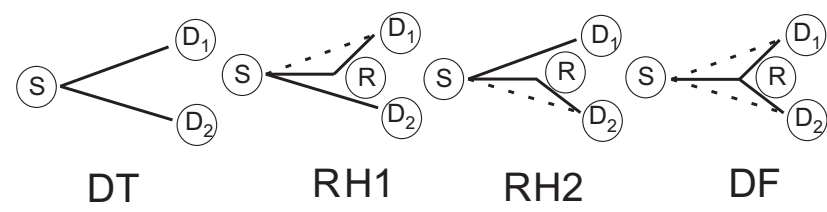

Fig. 3. Operation modes for PS and LCPS: direct transmission (DT), relay helps user 1 (RH1), relay helps user 2 (RH2) and decode-and-forward (DF). In PS, the destinations do not listen to the links shown with dashed lines, whereas in LCPS they do.

In LCPS, in the RH1 mode, if $b>a_{2}$, the source superimposes $X_{1}$ on $X_{2}$, and the opposite is true if $b<a_{2}$. Then the source sets $\left(\hat{P}_{S 1}^{(R H 1)}, \hat{P}_{S 2}^{(R H 1)}\right)=g\left(b, a_{2}, t\right)$ as in the RH1 mode of PS. However, in the RH1 mode of LCPS, $\mathrm{D}_{1}$ also listens to the source transmission. Then the achievable rate at $\mathrm{D}_{1}$ is

$$
C_{1}^{(R H 1)}=\left\{\begin{array}{l}
t \log \left(1+a_{1} \hat{P}_{S 1}^{(R H 1)}\right) \\
+(1-t) \log \left(1+c_{1} P_{R 1}\right) \\
t \log \left(1+\frac{a_{1} \hat{P}_{S 1}^{(R H 1)}}{a_{1} \hat{P}_{S 2}^{(R H 1)}+1}\right) \\
+(1-t) \log \left(1+c_{1} P_{R 1}\right)
\end{array} \quad\right. \text { otherwise }
$$

when $P_{R 1}$ denotes the relay power allocated to convey $W_{1}$. Solving $C_{1}^{(R H 1)}=R_{1}$ for $P_{R 1}$, we obtain the required relay power $\hat{P}_{R 1}^{(R H 1)}$ for reliable reception at $\mathrm{D}_{1}$. As the relay does not help $\mathrm{D}_{2}, \hat{P}_{R 2}^{(R H 1)}=0$. The required power levels for the $\mathrm{RH} 2$ mode are similarly obtained.

Suppose $P_{S j}$ and $P_{R j}$ are respectively the source and the relay power levels assigned to communicate $W_{j}$ with the $j$ th destination, $j=1,2$. In the DF mode, the relay has to decode both $W_{1}$ and $W_{2}$. The achievable rate for $W_{j}$ at the relay, $C_{S R j}^{(D F)}$, is then given as

$$
C_{S R j}^{(D F)}=\left\{\begin{array}{lll}
t \log \left(1+b P_{S j}\right) & \text { if } & a_{j}>a_{l} \\
t \log \left(1+\frac{b P_{S j}}{b P_{S l}+1}\right) & \text { if } & a_{j}<a_{l} .
\end{array}\right.
$$

for $j, l=1,2, j \neq l$, must be as large as the target rate $R_{j}$. Upon successful decoding, the relay forwards both messages. Then, the achievable rate at $\mathrm{D}_{j}$ is given by

$$
C_{j}^{(D F)}=C_{S j}^{(D F)}+C_{R j}^{(D F)},
$$

where

$$
\begin{aligned}
& C_{S j}^{(D F)}=\left\{\begin{array}{lll}
t \log \left(1+a_{j} P_{S j}\right) & \text { if } & a_{j}>a_{l} \\
t \log \left(1+\frac{a_{j} P_{S j}}{a_{j} P_{S l}+1}\right) & \text { if } & a_{j}<a_{l}
\end{array}\right. \\
& C_{R j}^{(D F)}= \begin{cases}(1-t) \log \left(1+c_{j} P_{R j}\right) & \text { if } \quad c_{j}>c_{l} \\
(1-t) \log \left(1+\frac{c_{j} P_{R j}}{c_{j} P_{R l}+1}\right) & \text { if } \quad c_{j}<c_{l}\end{cases}
\end{aligned}
$$

for $j, l=1,2, j \neq l$. Solving for $P_{S 1}, P_{S 2}, P_{R 1}$ and $P_{R 2}$ in $C_{1}^{(D F)}=C_{S R 1}^{(D F)}=R_{1}$ and $C_{2}^{(D F)}=C_{S R 2}^{(D F)}=R_{2}$, we obtain the required power levels $\hat{P}_{S 1}^{(D F)}, \hat{P}_{S 2}^{(D F)}, \hat{P}_{R 1}^{(D F)}$ and $\hat{P}_{R 2}^{(D F)}$. Substituting these required power levels for all modes 


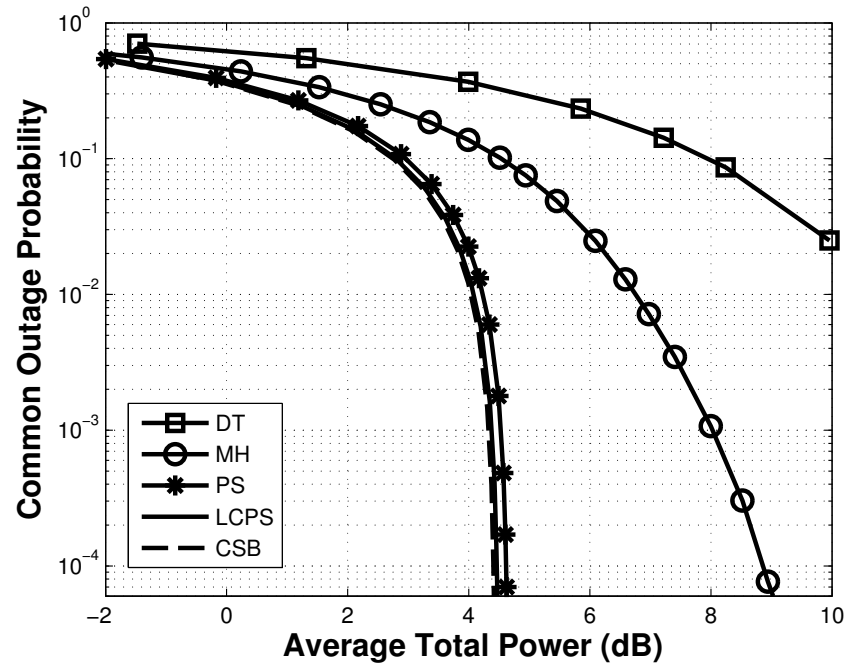

Fig. 4. The minimum common outage probability vs average total power where $R_{1}=1 R_{2}=1, d=0.2, d_{1}=0.25, d_{2}=0.25, \alpha=4$

of LCPS in (18) we then compute (6). Note that $\boldsymbol{A}_{L C P S}^{(i)}=$ $\boldsymbol{A}_{P S}^{(i)}, i=\mathrm{RH} 1, \mathrm{RH} 2, \mathrm{DF}$ and DT.

\section{E. The Cut-Set Bound}

In this section, we write the cut-set bound for the BRC [18]. The cut-set bound leads into two different upper bounds on the achievable rate pairs at $\mathrm{D}_{1}$ and $\mathrm{D}_{2}$.

In the first bound, we assume the relay is given the source messages $W_{1}$ and $W_{2}$ for free, or equivalently the relay can always decode $W_{1}$ and $W_{2}$ reliably. Then the system becomes similar to parallel broadcast channels, for which the capacity region is given in [12]. Using the results in [12], for a fixed $t$, achievable rates at $\mathrm{D}_{1}$ and $\mathrm{D}_{2}$ can respectively be upper bounded by $C_{1}=C_{1}^{(D F)}$ and $C_{2}=C_{2}^{(D F)}$ defined in (21).

The multiple antenna broadcast channel with two antennas at each destination and a single antenna at the source constitutes the second upper bound on the BRC under study. Using the capacity region results for the multiple antenna broadcast channel [19], we can upper bound the achievable rates at $\mathrm{D}_{1}$ and $\mathrm{D}_{2}$ for a fixed $t$ with

$$
\begin{aligned}
& \tilde{C}_{1}=\left\{\begin{array}{lll}
t \log \left(1+\left(a_{1}+b\right) P_{S 1}\right) & \text { if } & a_{1} \geq a_{2} \\
t \log \left(1+\frac{\left(a_{1}+b\right) P_{S 1}}{\left(a_{1}+b\right) P_{S 2}+1}\right) & \text { if } & a_{1}<a_{2}
\end{array}\right. \\
& \tilde{C}_{2}=\left\{\begin{array}{lll}
t \log \left(1+\frac{\left(a_{2}+b\right) P_{S 2}}{\left(a_{2}+b\right) P_{S 1}+1}\right) & \text { if } & a_{1} \geq a_{2} \\
t \log \left(1+\left(a_{2}+b\right) P_{S 2}\right) & \text { if } & a_{1}<a_{2} .
\end{array}\right.
\end{aligned}
$$

Combining the two bounds, achievable rates at $\mathrm{D}_{1}$ and $\mathrm{D}_{2}$ are respectively upper bounded by $\min \left(C_{1}, \tilde{C}_{1}\right)$ and $\min \left(C_{2}, \tilde{C}_{2}\right)$. Solving $\min \left(C_{1}, \tilde{C}_{1}\right)=R_{1}$ and $\min \left(C_{2}, \tilde{C}_{2}\right)=$ $R_{2}$ for $P_{S 1}, P_{S 2}, P_{R 1}$ and $P_{R 2}$, we calculate $\hat{P}_{S 1}^{(C S B)}(s)$, $\hat{P}_{S 2}^{(C S B)}(s), \hat{P}_{R 1}^{(C S B)}(s)$ and $\hat{P}_{R 2}^{(C S B)}(s)$ to obtain $P_{r e q}^{(C S B)}(s)$. Then we use $P_{r e q}^{(C S B)}(s)$ to obtain the minimum common outage probability defined in (6). This minimum common outage probability $P_{\text {out-min }}^{(C S B)}$ is a lower bound on all other protocols DT, MH, PS and LCPS. Based on the upper bounds

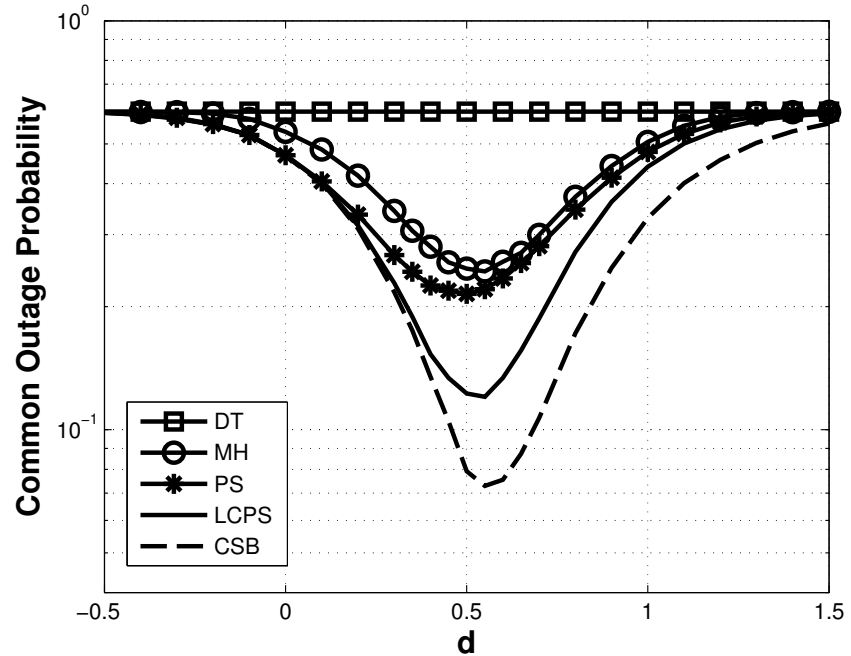

Fig. 5. The minimum common outage probability vs. relay location for $P_{a v g}$ $=0.5 \mathrm{~dB}, R_{1}=1, R_{2}=1, d_{1}=0.25, d_{2}=0.25$ and $\alpha=4$.

on achievable rates, $\min \left(C_{1}, \tilde{C}_{1}\right)$ and $\min \left(C_{2}, \tilde{C}_{2}\right)$, we also compute an upper bound on the $\epsilon$-outage rate region in the next section.

\section{NUMERICAL RESULTS}

Fig. 4 illustrates the minimum common outage probability vs. average total power for all protocols for $R_{1}=1 R_{2}=1, d$ $=0.2, d_{1}=0.25, d_{2}=0.25$ and $\alpha=4$. We observe that $\mathrm{MH}$ requires approximately $3 \mathrm{~dB}$ less total average power with respect to DT at $P_{\text {out }}=10^{-1}$, yet it is far from optimal. On the other hand, PS significantly improves upon MH and is only $0.2 \mathrm{~dB}$ away from the lower bound. This shows that enforcing the relay to help both destinations simultaneously is quite limiting, and path selection is necessary. As PS performs very close to LCPS, we can say that the gains obtained are mainly due to path selection rather than link combination at the destinations when $d=0.2$. This implies that simple receivers are sufficient when the relay is close to the source.

To see the effect of relay location on the performance, we plot the minimum common outage probability vs. relay location, $d$, in Fig. 5, for fixed average power $P_{\text {avg }}=0.5 \mathrm{~dB}, R_{1}$ $=1, R_{2}=1, d_{1}=0.25, d_{2}=0.25$ and $\alpha=4$. We observe that, when the relay is close to the source, PS is sufficient to attain the optimal behavior and link combination is not necessary. However, when the relay moves away from the source, the effect of link combination becomes emphasized. Optimum relay location for minimum common outage probability for the BRC is around $d=0.55$ for PS and LCPS, and $d=0.6$ for $\mathrm{MH}$.

Fig. 6 shows the $\epsilon$-outage rate region for $R_{1}$ and $R_{2}$ for a fixed minimum common outage probability of $0.01, P_{a v g}=1$ $\mathrm{dB}, d=0.3, d_{1}=0.2, d_{2}=0.4$ and $\alpha=4$. It can be seen that $\mathrm{MH}$ achieves a much larger $\epsilon$-outage rate region with respect to DT and the $\epsilon$-outage rate regions achievable with PS and LCPS are very close to the upper bound. We conclude that allowing the relay to help each of the destinations individually 


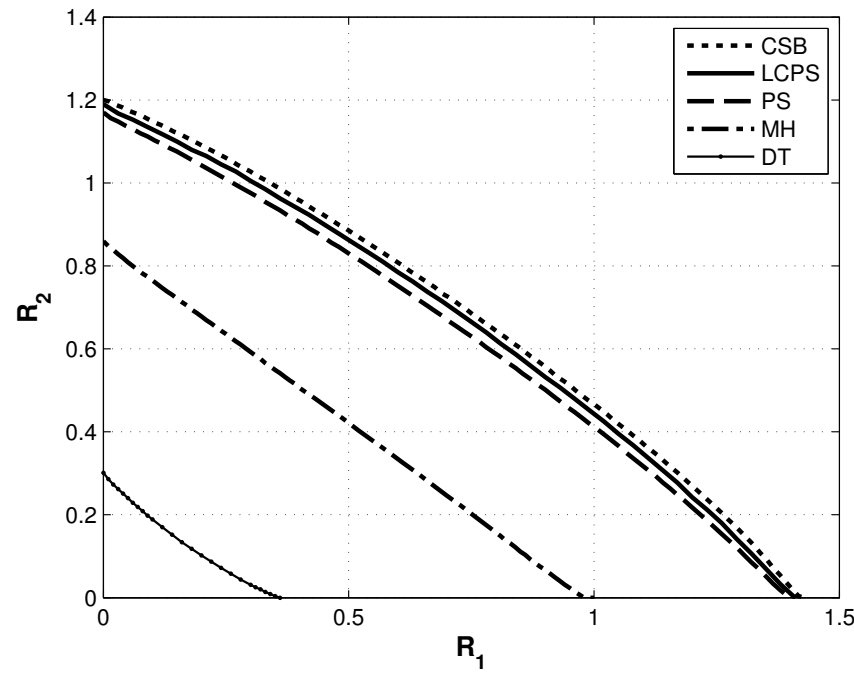

Fig. 6. The $\epsilon$-outage rate region for fixed minimum common outage probability of $0.01, P_{a v g}=1 \mathrm{~dB}, d=0.3, d_{1}=0.2, d_{2}=0.4$ and $\alpha=$

is almost optimal without the need of link combination at the destinations, when the relay is close to the source.

Finally, in Fig. 7, we plot the expected values of the ratios

$\zeta \triangleq \frac{\hat{P}_{S 1}^{(L C P S)}}{\hat{P}_{S 1}^{(L C P S)}+\hat{P}_{S 2}^{(L C P S)}}, \quad \beta \triangleq \frac{\hat{P}_{R 1}^{(L C P S)}}{\hat{P}_{R 1}^{(L C P S)}+\hat{P}_{R 2}^{(L C P S)}}$

for $P_{a v g}=0.5 \mathrm{~dB}, R_{1}=1, R_{2}=1, d_{1}=0.5$, and $\alpha=4$. We observe that, when $\mathrm{D}_{2}$ is colocated with $\mathrm{D}_{1}\left(d_{2}=-0.5\right)$, both the source and the relay allot their power equally among the two users, and $\mathbf{E}\{\zeta\}=\mathbf{E}\{\beta\}=0.5$. When $-0.5<d_{2}<0.5$, $\mathbf{E}\{\xi\}, \mathbf{E}\{\beta\}>0.5$. This is because the mean values for $a_{2}$ and $c_{2}$ are larger than the mean values for $a_{1}$ and $c_{1}$. Therefore, both the source and the relay allocate more power for $D_{1}$ to decrease the common outage probability. The opposite is true for $d_{2}>0.5$. It can be seen that $\mathbf{E}\{\zeta\}$ and $\mathbf{E}\{\beta\}$ reach their maximum values for $d_{2}=0$. In addition to these, we observe that the relay location $d$ has limited effect on $\mathbf{E}\{\zeta\}$ curve, while $\mathbf{E}\{\beta\}$ curve is highly dependent on the relay location.

\section{CONCLUSION}

In this paper, we study the four-terminal broadcast relay channel in terms of the minimum common outage probability under long-term power constraint and constant rate transmission. We propose four different protocols, direct transmission, multihop, path selection and link combination with path selection. We also find the $\epsilon$-outage rate region for all protocols, and compare the performances of all four protocols with the cutset bound. Our results indicate that enforcing the relay to help both destinations simultaneously is limiting. When the relay is close to the source, path selection is sufficient for optimal behavior, whereas link combination becomes necessary to obtain additional gains, when the source-relay distance grows larger. Future work includes investigating the amplify-andforward protocol, non-orthogonal relaying, and the outage probability region when outage is declared individually for each of the destinations.

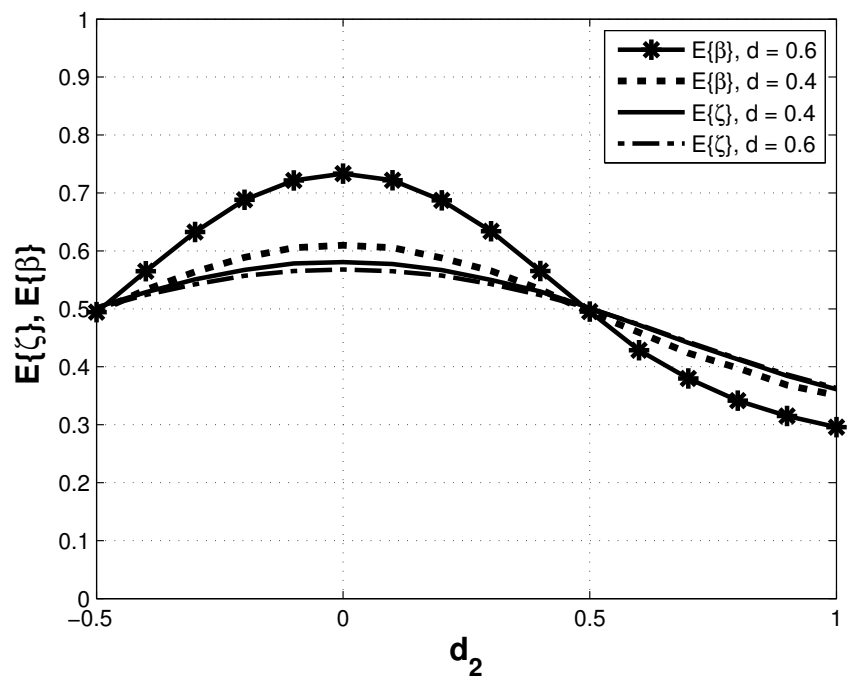

Fig. 7. $\mathbf{E}\{\zeta\}$ and $\mathbf{E}\{\beta\}$ (defined in (24)) vs. $d_{2}$ for $P_{a v g}=0.5 \mathrm{~dB}, R_{1}=$ $1, R_{2}=1, d_{1}=0.5$ and $\alpha=4$ for LCPS.

\section{REFERENCES}

[1] T. M. Cover and A. E. Gamal, "Capacity theorems for the relay channel," IEEE IT, vol. 25, p. 572, September 1979.

[2] A. Sendonaris, E. Erkip, and B. Aazhang, "User cooperation diversityPart I, Part II," IEEE Transactions on Communications, vol. 51, pp. 1927-1948, November 2003.

[3] J. N. Laneman, D. N. C. Tse, and G. W. Wornell, "Cooperative diversity in wireless networks: Efficient protocols and outage behavior," IEEE IT, vol. 50, p. 3062, December 2004.

[4] IEEE IT, Special Issue on Models, Theory, and Codes for Relaying and Cooperation in Communication Networks, October 2007.

[5] IEEE TWC, Special Issue on Cooperative Communication, May 2008.

[6] IEEE JSAC, Special Issue on Cooperative Communication and Networking, February 2007.

[7] G. Kramer, M. Gastpar, and P. Gupta, "Cooperative strategies and capacity theorems for relay networks," IEEE IT, vol. 51, no. 9, p. 3037, 2005.

[8] S. Bhaskaran, "Gaussian degraded relay broadcast channel," IEEE IT, vol. 54, pp. 3699-3709, August 2008.

[9] A. Behboodi and P. Piantanida, "Capacity of a class of broadcast relay channels," in Proc. IEEE ISIT 2010, pp. 590 -594, June, 13-18 2010.

[10] C. Liang, "On rate region bounds of broadcast relay channels," in Proc. 44th CISS 2010.

[11] G. Caire, G. Taricco, and E. Biglieri, "Optimum power control over fading channels," IEEE IT, vol. 45, pp. 1468 -1489, July 1999.

[12] D. Tse, "Optimal power allocation over parallel Gaussian broadcast channels.".

[13] L. Li and A. Goldsmith, "Capacity and optimal resource allocation for fading broadcast channels .ii. outage capacity," IEEE IT, vol. 47, pp. $1103-1127$, March 2001.

[14] M. Hasna and M.-S. Alouini, "Optimal power allocation for relayed transmissions over Rayleigh-fading channels," IEEE TWC, vol. 3, pp. 1999 - 2004, November 2004.

[15] A. Høst-Madsen and J. Zhang, "Capacity bounds and power allocation for wireless relay channels," IEEE IT, vol. 51, p. 2020, June 2005.

[16] D. Gunduz and E. Erkip, "Opportunistic cooperation by dynamic resource allocation," IEEE TWC, vol. 6, pp. 1446 -1454, April 2007.

[17] Y. Liang, V. Veeravalli, and H. Poor, "Resource allocation for wireless fading relay channels: Max-min solution," IEEE IT, vol. 53, pp. 3432 -3453 , October 2007.

[18] T. M. Cover and J. A. Thomas, Elements of Information Theory. JohnWiley \& Sons, Inc., 1991.

[19] H. Weingarten, Y. Steinberg, and S. Shamai, "The capacity region of the Gaussian multiple-input multiple-output broadcast channel," IEEE IT, vol. 52, pp. 3936 -3964, September 2006. 